\title{
Procesos de cambio en conductas adictivas: Influencia de variables psicopatológicas y de consumo
}

\author{
Sánchez-Hervás, E.; Tomás Gradolí, V.; Molina Bou, N.; Del Olmo Gurrea, R.; Morales Gallús, E. \\ Unidad de Conductas Adictivas Area 9. Catarroja. Consellería de Sanitat. Valencia. \\ Enviar correspondencia: \\ Emilio Sánchez Hervás. Unidad de Conductas Adictivas. Centro de Salud de Catarroja. Avda. Rambleta s/n. 46470 Catarroja (Valencia). \\ E-mail: uca.catarroja@trabajo.m400.gva.es
}

\section{RESUMEN}

Considerando los niveles de procesos de cambio del modelo de Prochaska y DiClemente y asumiendo la influencia en dichos procesos de otras variables presentes en los pacientes cuando demandan tratamiento, nos propusimos estudiar la posible relación entre la aparición de los diferentes procesos de cambio, la presencia de psicopatología y las variables de la historia toxicológica de los sujetos (años de consumo, años de abuso y tratamientos previos).

Se estudian 107 pacientes divididos en tres grupos (grupo de dependencia a alcohol $n=35$, grupo dependencia a heroína $n=34$, grupo dependencia a cocaína $n=38$ ). La información se recoge a través de entrevista al ingreso y aplicando el Inventario de Procesos de Cambio y el Brief Symptom Inventory. Los resultados indican que independientemente del diagnóstico de los pacientes, se utilizan más procesos de cambio cognitivos cuando se produce la demanda de tratamiento y en menor medida procesos de cambio de tipo conductual. La psicopatología y las variables de consumo influyen en la utilización de determinados procesos de cambio.

Podría argumentarse que la presencia de distress emocional y la sintomatología influyen en mayor medida en los procesos de tipo cognitivo, mientras que el historial de uso y abuso de las sustancias provocarían mayores dificultades para adquirir y desarrollar estrategias conductuales de cambio.

Sugerímos que la intervención al inicio de los tratamientos se centre en la educación de los pacientes para el manejo de la sintomatología negativa y el distress emocional, mediante la utilización de técnicas de counseling, que permitieran una mayor estabilidad psicológica en los pacientes y una mayor adherencia al tratamiento.

Palabras clave: alcohol, heroína, cocaína, motivación, psicopatología, drogodependencia.

\section{ABSTRACT}

Taking into account the levels of the stages of change in the Prochaska and DiClemente model, and assuming the influence of other variables present in patients when they demand treatment on said stages, we proposed studying the possible relationship between the appearance of the different stages of change, the presence of psychopathology and the variables of the patient's toxicological history (years of use, years of abuse and previous treatments).

107 patients were studied, divided into three groups (alcohol-dependent group $n=35$, heroin-dependent group $n=34$ and cocaine-dependent group $n=38$ ). The information was obtained from an interview on admission and by applying the Stages of Change and the Brief Symptom Inventory.

The results show that, independently of the diagnosis of the patients, more stages of cognitive change are used when the demand for treatment occurs and, to a lesser extent, behaviour type stages of change. Use psychopathology and the use variables influence the utilisation of certain processes of change.

It could be argued that the presence of emotional distress and the symptomology have a greater influence on cognitivetype processes whereas the history of substance use and abuse would provoke greater difficulties in acquiring and developing behaviour change strategies.

We suggest that intervention at the commencement of treatment should be centred on the education of patients in order for them to be able to handle the negative symptomology and emotional distress by the use of counselling techniques that would allow a greater psychological stability in patients and a greater adherence to treatment.

Key words: alcohol, heroin, cocaine, motivation, psychopathology, drug addiction.

cias como la heroína y la cocaína), han supuesto la proliferación de diversos enfoques para explicar la naturaleza de tales problemas y, las estrategias terapéuticas para tratarlos. En una revisión realizada por Lettiere, Seyers y Pearson (1980), se identifican más 
de cuarenta perspectivas teóricas que tratan de explicar los problemas y las conductas de adicción a sustancias de abuso.

Hasta la década de los ochenta la explicación de las conductas adictivas se fundamentaba en el modelo moral y en el modelo biológico (Marlatt, 1985), aunque surgieron distintos posicionamientos teóricos con mayor o menor éxito: modelos socioculturales (Bacon 1974; Jones, 1975; Bell, 1975), teorías psicodinámicas (Cutter, 1973; Schukit, 1976) y transaccionales (Steiner, 1971), teorías de la personalidad (Blum, 1966, Vaillant, 1983), etc.

En los años ochenta comienzan a parecer diversos trabajos que enfatizan un modelo de fases de cambio en la descripción, explicación y predicción del proceso de abandono de diferentes conductas adictivas, en contraposición a la visión tradicional del cambio como un suceso dicotómico (abstinencia vs consumo). La ventaja de este tipo de modelos es que describen el cambio como un proceso a lo largo del tiempo, en lugar de enfatizar la abstinencia "per se" como el primer objetivo a alcanzar (Tejero y Trujols, 1994). El modelo transteorético de cambio de Prochaska y DiClemente (Prochaska y DiClemente, 1992, Prochaska DiClemente y Norcross, 1992, Prochaska y Prochaska, 1993) es el que ha recibido un mayor soporte en relación a su capacidad descriptiva y predictiva del proceso de cambio en las conductas adictivas.

El modelo transteorético de Prochaska y DiClemente delimita y describe los elementos subyacentes al proceso de cambio intencional de cualquier conducta adictiva. Se ha desarrollado a partir del análisis de las teorías y las investigaciones en psicoterapia y, en los trabajos de los autores en relación al proceso de cambio en las adicciones. Es un modelo tridimensional que proporciona una concepción global y diferenciada del proceso de cambio en el campo de las conductas adictivas, integrando tres elementos: estadios, procesos y niveles de cambio (Prochaska, DiClemente y Norcross, 1992). Los estadios representan la estructura o dimensión temporal evolutiva del modelo: precontemplación, contemplación, preparación, acción y mantenimiento.

El segundo nivel del modelo lo forman los procesos de cambio, éstos permiten conocer cómo se producen los cambios en los sujetos. Consisten en actividades manifiestas o encubiertas iniciadas o experimentadas por un individuo para modificar su hábito adictivo. Prochaska y DiClemente señalan que estos diez procesos de cambio se estructuran en relación a dos factores de segundo orden: a) procesos de cambio cognitivos: aumento de la concienciación, autorreevaluación, reevaluación ambiental, relieve dramático y liberación social; b) procesos de cambio conductuales: autoliberación, manejo de contingencias, relaciones de autoayuda, contracondicionamiento y control de estímulos.
Los estadios y procesos de cambio tienen que situarse en el contexto de los niveles complejos e interrelacionados del comportamiento humano. La tercera dimensión del modelo hace referencia a qué cambios se necesitan para abandonar la conducta adictiva. Esta tercera dimensión denominada de los niveles de cambio representa una organización jerárquica de los cinco niveles en que se focalizan las diferentes evaluaciones e intervenciones terapeúticas: síntoma/situacional, cogniciones desadaptadas, conflictos interpersonales actuales, conflictos sistémicos/familiares, y conflictos intrapersonales.

El trabajo que presentamos a continuación es un intento de plasmar las impresiones clínicas de los autores en su práctica diaria. Considerando el nivel de procesos de cambio del modelo de Prochaska y DiClemente y asumiendo la influencia en dichos procesos de otras variables presentes en los pacientes cuando demandan tratamiento, nos propusimos estudiar la posible relación entre la aparición de los diferentes procesos de cambio, la presencia de psicopatología y las variables de la historia toxicológica de los sujetos (años de consumo, años de abuso y tratamientos previos), así cómo las implicaciones que tales procesos pudieran tener sobre las intervenciones terapéuticas.

\section{MATERIAL Y METODO}

\section{Muestra}

La muestra la componen 107 sujetos, pacientes de la Unidad de Conductas Adictivas de Catarroja (Area 9 de Salud -Valencia-) que demandaron tratamiento durante el año 1999-2000 y distribuidos en función del diagnóstico realizado según criterios DSM-IV (APA, 1995): Grupo dependencia a alcohol $(n=35)$; grupo dependencia a heroína $(n=34)$ y, grupo dependencia a cocaína ( $n=38)$.

Criterios de inclusión: dependencia a alcohol (grupo A), dependencia a heroína (grupo B) y dependencia a cocaína (grupo C). Haber superado con éxito la desintoxicación, en aquellos casos en que fue necesaria.

Criterios de exclusión: analfabetismo, no completar las pruebas.

\section{Diseño}

Estudio transversal

\section{Instrumento}

1. Entrevista estructurada: utilizada para todos los pacientes del servicio. En el trabajo se utilizan 
las variables edad, sexo, situación laboral y nivel de estudios, tratamientos previos, años de consumo y años de abuso de la sustancia objeto de la demanda, todas ellas extraídas de la entrevista. Se administra en las primeras sesiones con información aportada por el paciente y por los acompañantes.

2. Para la evaluación de la psicopatología se utilizó el Brief Symptom Inventory (BSI) de Derogatis (1975): instrumento de screening; forma abreviada del SCL-90 del que también es autor Derogatis $y$, del que posteriormente apareció una versión revisada, el SCL-90-R (Derogatis, 1983). EI BSI tiene una buena correlación con el SCL90, por encima de 0.90 en todas las dimesiones (Derogatis y Cleary, 1977), y además es mucho más ágil en su administración (53 ítems), lo que facilita su administración en usuarios de drogas. Incluye (al igual que el SCL-90) nueve dimensiones sintomáticas: somatización, obsesión-compulsión, sensibilidad interpersonal, depresión, ansiedad, hostilidad, ansiedad fóbica, ideación paranoide y, psicoticismo. Además de estas nueve dimensiones, se incluyen tres índices globales que describen la intensidad de la psicopatología del encuestado: índice de severidad global, índice de malestar y, total de síntomas positivos. El índice de severidad global (GSI) proporciona un indicador sensible de la respuesta del nivel de estrés experimentado por el paciente. Combina la información del número de síntomas presentes y la intensidad del distress. El índice de malestar (PSDI) es una medida de la intensidad del nivel de distress esperimentado en función del número de síntomas, informando sobre el estilo de respuesta de distress. Finalmente, el indicador total de síntomas positivos (PST) revela el número de síntomas que el paciente afirma experimentar $y$, se utiliza conjuntamente con los otros indicadores globales comunicando la amplitud del distress emocional del individuo. En el estudio que se presenta se utilizan estos tres indicadores globales de psicopatología. La razón de ello es que los indicadores globales han demostrado ser una buena medida de malestar psicológico general tanto en la población normal (Aragón y col, 2000), como en muestras clínicas (Boulet y Boss, 1991; Piersma, 1994).

3. Inventario de Procesos de Cambio (Tejero, Trujols y Hernandez, 1990; Tejero y Trujols, 1994). Autoinforme de 40 ítems (cuatro para cada una de las diez escalas correspondientes a los diez procesos de cambio) desarrollado conforme a las directrices teóricas que se desprenden del trabajo de Prochaska y DiClemente, y basado en el inventario de procesos de cambio desarrollado por estos autores para fumadores (DiClemente y Prochaska, 1985). Consta de una escala Likert de cuatro puntos que mide la frecuencia con la que se utilizan las distintas estrategias cognitivo-conductuales descritas para cada uno de los ítems. Las puntuaciones en cada proceso oscilan entre 0 y 12. La finalidad del instrumento es la de identificar qué procesos de cambio ha desarrollado un determinado paciente (y conocer por tanto en qué estadio de cambio se encuentra). De esta forma el terapeuta puede conocer que estrategias puede utilizar con el paciente, para que éste desarrolle los procesos de cambio que le permitan progresar hacia el próximo estadio, en definitiva hacia el mantenimiento de la abstinencia (Tejero y Trujols, 1994). Los autores han utilizado una versión de dicho instrumento para los pacientes del grupo alcohol y del grupo cocaína, modificando las expresiones que hacían referencia a la sustancia objeto de abuso (heroína en el original) por las de alcohol y cocaína. En el anexo 1 se muestra el cuestionario utilizado.

\section{Variables}

Variables sociodemográficas: edad, sexo, nivel de estudios, situación laboral. Obtenidas de las historias clínicas de los pacientes.

Variables clínicas: puntuaciones en el Inventario de Procesos de Cambio.

Variables psicométricas: índices globales del BSI (GSI, PSDI, PST), años de abuso, años de consumo y tratamientos anteriores.

\section{Análisis de datos}

Para la descripción de la muestra, y para la estimación de las puntuaciones en los instrumentos de evaluación se han realizado análisis de frecuencias y medidas de tendencia central. Para el contraste de diferencias se realizaron análisis de varianza y prueba de Scheefé para la comparación de los grupos a posteriori. Para la correlación de variables se utilizó el coeficiente de correlación de Pearson. En la interpretación de resultados se eligió un nivel de significación estadística de $\mathrm{p}<0.05$, para un intervalo de confianza del $95 \%$.

\section{RESULTADOS}

En la tabla 1 aparecen los resultados obtenidos al analizar las variables descriptivas de la muestra.

Los resultados muestran que la edad media del grupo de alcohol $(37,7)$ es mayor que la del grupo de 


\title{
ANEXO 1. INVENTARIO DE PROCESOS DE CAMBIO (Tejero y Trujols, 1994)
}

\author{
(Modificado)*
}

Instrucciones: a continuación encontrarás una serie de comentarios referentes a conductas, pensamientos y sensaciones de algunos adictos y ex-adictos. Léelos atentamente e indica utilizando el número de la escala que encontrarás a continuación la frecuencia con que últimamente te ocurren cosas que en ellos se describen.

0 No es en absoluto mi caso; 1 Algunas veces es mi caso; 2 Bastantes veces es mi caso; 3 Muchas veces es mi caso.

$\begin{array}{llll}0 & 1 & 2 & 3\end{array}$

0

$\begin{array}{llll}0 & 1 & 2 & 3 \\ 0 & 1 & 2 & 3\end{array}$

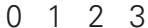

$\begin{array}{llll}0 & 1 & 2 & 3\end{array}$

$\begin{array}{llll}0 & 1 & 2 & 3\end{array}$

$\begin{array}{llll}0 & 1 & 2 & 3\end{array}$

$\begin{array}{llll}0 & 1 & 2 & 3\end{array}$

$\begin{array}{llll}0 & 1 & 2 & 3\end{array}$

$\begin{array}{llll}0 & 1 & 2 & 3\end{array}$

$\begin{array}{llll}0 & 1 & 2 & 3\end{array}$

$\begin{array}{llll}0 & 1 & 2 & 3\end{array}$

$\begin{array}{llll}0 & 1 & 2 & 3\end{array}$

$\begin{array}{llll}0 & 1 & 2 & 3\end{array}$

$\begin{array}{llll}0 & 1 & 2 & 3\end{array}$

$\begin{array}{llll}0 & 1 & 2 & 3\end{array}$

$\begin{array}{llll}0 & 1 & 2 & 3\end{array}$

$\begin{array}{llll}0 & 1 & 2 & 3\end{array}$

$\begin{array}{llll}0 & 1 & 2 & 3\end{array}$

$\begin{array}{llll}0 & 1 & 2 & 3\end{array}$

$\begin{array}{llll}0 & 1 & 2 & 3\end{array}$

$\begin{array}{llll}0 & 1 & 2 & 3\end{array}$

$\begin{array}{llll}0 & 1 & 2 & 3\end{array}$

$\begin{array}{llll}0 & 1 & 2 & 3\end{array}$

$\begin{array}{llll}0 & 1 & 2 & 3\end{array}$

$\begin{array}{llll}0 & 1 & 2 & 3\end{array}$

$\begin{array}{llll}0 & 1 & 2 & 3\end{array}$

$\begin{array}{llll}0 & 1 & 2 & 3\end{array}$

$\begin{array}{llll}0 & 1 & 2 & 3\end{array}$

$\begin{array}{llll}0 & 1 & 2 & 3\end{array}$

$\begin{array}{llll}0 & 1 & 2 & 3\end{array}$

$\begin{array}{llll}0 & 1 & 2 & 3\end{array}$

$\begin{array}{llll}0 & 1 & 2 & 3\end{array}$

$\begin{array}{llll}0 & 1 & 2 & 3\end{array}$

$\begin{array}{llll}0 & 1 & 2 & 3\end{array}$

$\begin{array}{llll}0 & 1 & 2 & 3\end{array}$

$\begin{array}{llll}0 & 1 & 2 & 3\end{array}$

$\begin{array}{llll}0 & 1 & 2 & 3\end{array}$

$\begin{array}{llll}0 & 1 & 2 & 3\end{array}$

$\begin{array}{llll}0 & 1 & 2 & 3\end{array}$
1. Voy comprometiéndome a no tomar droga

2. Me molesta ser rechazado por los demás por el hecho de ser drogodependiente

3. Existen personas en mi vida diaria que se preocupan de que me sienta bien cuando no tomo droga

4. Ver a otros drogodependientes en pésimas condiciones físicas me hace sentir mal

5. Me siento a disgusto cuando pienso en mi problema con la droga

6. Pienso que mi vida familiar, afectiva o amorosa sería mejor si no tomase drogas

7. Me viene a la cabeza el recuerdo de artículos de periódico o programas de televisión o radio que hablan sobre los problemas con los que un drogadicto se encuentra al dejar la droga

8. A través de los medios de comunicación, conozco las campañas que se están llevando a cabo contra la droga

9. Creo que los drogadictos podemos llegar a ser personas tan útiles para la sociedad como cualquier otra persona si dejamos nuestra adicción

10. Pienso en la información que la gente me ha dado sobre los beneficios de dejar la droga

11. Sé que cada vez más la gente piensa que ser drogadicto significa ser delincuente o un enfermo de Sida

12. Quito de en medio cosas de mi casa que me provocan ganas de tomar droga

13. Cuando tengo ganas de tomar droga realizo alguna actividad física para que desaparezcan

14. Mi dependencia de la heroína me hace sentirme a disgusto conmigo mismo

15. Suelo abrirme o sincerarme con alguna persona para explicarle mis experiencias con la droga

16. Pienso en la información de artículos y anuncios del periódico o programas de televisión que hablan sobre como uno puede dejar de tomar drogas

17. Creo que el que yo tome droga perjudica a las personas que me rodean

18. Acostumbro a poner alrededor de los lugares en donde paso más horas (casa, habitación, trabajo, bar, etc), cosas que me recuerdan que no debo tomar drogas

19. El recordar que tomar drogas produce diversas enfermedades o problemas de salud, me afecta emocionalmente (me siento nervioso, preocupado, etc).

20. Evito ir a divertirme a lugares donde sé que van personas que consumen drogas

21. Para no tomar drogas, me vuelvo por barrios en donde no puedo encontrarla

22. Hay algunas personas especiales en mi vida que me aceptan como lo que soy, una persona, tanto si me drogo como si no me drogo

23. Me digo a mi mismo que soy capaz de dejar la droga si así lo quiero

24. Sé que los demás piensan que si tomo droga es porque soy una persona peligrosa y crea problemas, pero yo sé que no es cierto, y que quiero dejar la droga para demostrarlo

25. Cada vez más la gente de mi ciudad es consciente del problema de las toxicomanías y se están movilizando para prevenirlas

26. Creo que el tomar drogas afecta las relaciones que tengo con las demás personas

27. Cuando estoy tentado de tomar droga, pienso en cualquier otra cosa que pueda distraerme

28. Me digo a mí mismo que puedo escoger entre tomar droga y no tomarla

29. Recuerdo la información que la gente me ha dado sobre cómo dejar la droga

30. Pienso que para poder estar bien conmigo mismo debo dejar de tomar droga

31. Me felicito, me recompenso o me regalo a mi mismo cuando no tomo droga

32. Cuando necesito relajarme para enfrentarme a mis tensiones o problemas, hago otras cosas en lugar de drogarme

33. Evito encontrarme con amigos que sé que están tomando droga

34. Me afecta emocionalmente (me siento tenso, preocupado, etc.) cuando me advierten de los problemas de relación (familiares, de pareja o de amistad) que supone el tomar droga

35. Sé que alguien me felicitará o me recompensará si no tomo droga

36. Tengo a alguien con quien puedo contar cuando tengo problemas con la droga

37. Me digo a mi mismo que si lo intento con bastante firmeza puedo estar sin tomar droga

38. Los consejos o advertencias sobre lo peligroso que resulta para la salud tomar droga me afecta emocionalmente (me siento nervioso, preocupado, etc.)

39. Tengo alguien que me escucha cuando necesito hablar sobre mi relación con la droga

40. Existen algunas personas que cuando no tomo droga me recompensan o me felicitan

* Para los pacientes dependientes a heroína se utilizó el cuestionario original, y para los dependientes a alcohol y cocaína se sustituyó el termino droga, por el de cocaína y alcohol. 
heroína $(29,1)$ y ésta a su vez mayor que la del grupo de cocaína $(26,1)$. En cuanto al sexo, la muestra la componen mayoritariamente hombres (alcohol 93,3\%; heroína 83,9\%; cocaína 90,7\%). En cuanto a la situación laboral los grupos alcohol y cocaína presentan resultados similares $175,9 \%$ del grupo de alcohol y 68,3\% del grupo de cocaína están activos laboralmente), mientras que el 72,4\% del grupo de heroína está parado. Los estudios primarios predomi- nan en todos los grupos: grupo alcohol (75\%), heroína $(83,3 \%)$ y cocaína $(76,9 \%)$. En general puede decirse que las características de nuestra muestra son similares a los datos de pacientes dependientes a alcohol y drogas ilegales recogidos por los organismos oficiales (PNSD, 1998, 1999).

En la tabla 2 aparecen los resultados del análisis de las variables de consumo y de psicopatología (indicado-

\begin{tabular}{|c|c|c|c|c|c|}
\hline & & $\begin{array}{c}\text { GRUPO ALCOHOL } \\
\mathrm{N}=35\end{array}$ & $\begin{array}{c}\text { GRUPO HEROÍNA } \\
\text { N=34 }\end{array}$ & $\begin{array}{c}\text { GRUPO COCAÍNA } \\
\mathrm{N}=38\end{array}$ & $\mathbf{P}$ \\
\hline EDAD & (Media-Dt) & $37,0(10,8)$ & $29,1(4,6)$ & $26,1(7,5)$ & $0.003^{\mathrm{ab}}$ \\
\hline SEXO & $\begin{array}{l}\text { Hombre } \\
\text { Mujer }\end{array}$ & $\begin{array}{c}93,3 \\
6,7 \\
\end{array}$ & $\begin{array}{l}83,9 \\
16,1\end{array}$ & $\begin{array}{c}90,7 \\
9,3\end{array}$ & NS \\
\hline SITUACIÓN LABORAL & $\begin{array}{l}\text { Activo } \\
\text { Parado }\end{array}$ & $\begin{array}{l}75,9 \\
24,1\end{array}$ & $\begin{array}{l}27,6 \\
72,4\end{array}$ & $\begin{array}{l}68,3 \\
31,7\end{array}$ & $0.002^{c}$ \\
\hline ESTUDIOS & $\begin{array}{l}\text { Primarios } \\
\text { Secundarios } \\
\text { Universitarios }\end{array}$ & $\begin{array}{c}75 \\
17,9 \\
7,1 \\
\end{array}$ & $\begin{array}{c}83,3 \\
16,7 \\
- \\
\end{array}$ & $\begin{array}{c}76,9 \\
20,5 \\
2,6\end{array}$ & NS \\
\hline
\end{tabular}

res globales del BSI). El grupo alcohol presenta mayor antigüedad en el consumo y más años de abuso (17,8 y 11,0 años), seguido del grupo heroína (10,4 y 8,3 años) y del grupo cocaína (7,4 y 4,3 años). Las diferencias en cuanto a los años de consumo son estadísticamente significativas entre el grupo alcohol y el grupo heroína y entre el grupo alcohol y el grupo cocaína. También aparecen diferencias significativas en la variable años de abuso entre el grupo alcohol y el grupo cocaína y entre el grupo heroína y el grupo cocaína. Finalmente el grupo heroína presenta mayor número de tratamientos que los grupos alcohol y cocaína, con diferencias estadísticamente significativas entre los grupos heroína y alcohol y los grupos heroína y cocaína.

\section{TABLA 2. VARIABLES TOXICOLÓGICAS Y PSICOPATOLÓGICAS}

\begin{tabular}{|l|c|c|c|c|}
\hline & $\begin{array}{c}\text { GRUPO ALCOHOL } \\
\mathbf{N = 3 5}\end{array}$ & $\begin{array}{c}\text { GRUPO HEROÍNA } \\
\mathbf{N = 3 4}\end{array}$ & $\begin{array}{c}\text { GRUPO COCAÍNA } \\
\mathbf{N = 3 8}\end{array}$ & P \\
\hline AÑOS DE CONSUMO & $17,8(10,3)$ & $10,4(9,1)$ & $7,4(7,1)$ & $0.000^{\mathrm{ab}}$ \\
AÑOS DE ABUSO & $11.0(7,5)$ & $8,3(6,3)$ & $4,3(4,5)$ & $0.000^{\mathrm{bc}}$ \\
TRATAMIENTOS ANTERIORES & $0,8(1,0)$ & $1,7(1,7)$ & $0,8(1,1)$ & $0.006^{\mathrm{ac}}$ \\
BSI-GSI & $1,0(0,8)$ & $0,8(0,6)$ & $0,8(0,5)$ & $\mathrm{NS}$ \\
BSI-PSDI & $1,9(0,9)$ & $1,7(0,6)$ & $1,6(0,6)$ & $\mathrm{NS}$ \\
BSI-PST & $26,2(10)$ & $25,0(11,7)$ & $23,7(11,4)$ & $\mathrm{NS}$ \\
\hline
\end{tabular}

Valores medios y desviación estándar de las variables toxicológicas y psicopatológicas. Comparación de medias mediante ANOVA. Comparaciones múltiples mediante prueba de Schefée. NS=No significativo. BSI=Brief Symptom Inventory, GSI=Indice de Severidad Global, PSDI=Indice de Malestar. PST=Total Síntomas Positivos. ${ }^{\circ} G r$.Alcohol vs Gr.Heroína, bGr.Alcohol vs Gr.Cocaína, 'Gr. Heroína vs Gr.Cocaína.

En la tabla 3 se muestran las puntuaciones obtenidas en el Inventario de Procesos de Cambio. Las puntuaciones más elevadas en el grupo alcohol aparecen en los procesos reevaluación ambiental $(9,7)$, autolibe- ración $(9,2)$, relieve dramático $(8,3)$ y autoreevaluación. En el grupo heroína destacan las puntuaciones en los procesos reevaluación ambiental $(9,5)$, autoliberación $(8,8)$ y autoreevaluación $(8,3)$. En el grupo cocaína los 
resultados son similares, mayores puntuaciones en los procesos reevaluación ambiental $(9,4)$, autoliberación $(8,7$ y relaciones de autoayuda $(8,2)$. Las puntuaciones más bajas corresponden en los tres grupos al proceso control de estímulos (3,9 grupo alcohol vs 4,3 grupo heroína vs 4,0 grupo cocaína). El análisis de varianza muestra que no existen diferencias estadísticamente significativas entre los grupos de estudio para ninguno de los procesos de cambio.
En la tabla 4 aparecen los resultados de las correlaciones entre los procesos de cambio, las variables de consumo y las variables psicopatológicas. Los años de consumo correlacionan positivamente con el proceso de liberación social y de forma inversa con el proceso contracondicionamiento. Los años de abuso correlacionan de forma inversa con los procesos contracondicionamiento, control de estímulos y relaciones de autoayuda. El índice de severidad global (GSI) correla-

\section{TABLA 3. PUNTUACIONES DE LOS PROCESOS DE CAMBIO MEDIDAS CON EL IPC}

\begin{tabular}{|c|c|c|c|c|c|}
\hline & & $\begin{array}{c}\text { GRUPO ALCOHOL } \\
\mathrm{N}=35\end{array}$ & \begin{tabular}{|c|}
$\begin{array}{c}\text { GRUPO HEROÍNA } \\
\mathrm{N}=34\end{array}$ \\
\end{tabular} & $\begin{array}{c}\text { GRUPO COCAÍNA } \\
\mathrm{N}=38\end{array}$ & $\mathbf{P}$ \\
\hline $\begin{array}{l}\text { IPC PROCESOS } \\
\text { COGNITIVOS }\end{array}$ & $\begin{array}{l}\text { Aumento de } \\
\text { Concienciación } \\
\text { Relieve Dramático } \\
\text { Reevaluación } \\
\text { Ambiental } \\
\text { Autorreevaluación } \\
\text { Liberación social }\end{array}$ & $\begin{array}{l}6,1(3,4) \\
8,3(3,1) \\
9,7(2,4) \\
8,3(3,1) \\
6,1(3,4)\end{array}$ & $\begin{array}{l}5,7(3,2) \\
7,3(3,9) \\
9,5(2,7) \\
8,3(2,9) \\
6,9(2,9)\end{array}$ & $\begin{array}{l}6,2(3,0) \\
6,7(3,4) \\
9,4(2,9) \\
7,7(3,0) \\
5,2(2,7)\end{array}$ & $\begin{array}{l}\text { NS } \\
\text { NS } \\
\text { NS } \\
\text { NS } \\
\text { NS }\end{array}$ \\
\hline $\begin{array}{l}\text { IPC PROCESOS } \\
\text { CONDUCTUALES }\end{array}$ & $\begin{array}{l}\text { Autoliberación } \\
\text { Manejo Contingenc. } \\
\text { Relac. de Autoayuda } \\
\text { Contracondicionam. } \\
\text { Control de Estímulos }\end{array}$ & $\begin{array}{l}9,2(2,9) \\
7,7(3,4) \\
7,6(3,1) \\
4,7(3,9) \\
3,9(3,7)\end{array}$ & $\begin{array}{l}8,8(2,8) \\
8,0(3,5) \\
7,1(2,9) \\
5,3(3,7) \\
4,3(3,9)\end{array}$ & $\begin{array}{l}8,7(3,1) \\
7,2(3,2) \\
8,2(3,1) \\
4,9(3,4) \\
4,0(2,6)\end{array}$ & $\begin{array}{l}\text { NS } \\
\text { NS } \\
\text { NS } \\
\text { NS } \\
\text { NS }\end{array}$ \\
\hline
\end{tabular}

Valores medios y desviación estándar de las variables de cambio. Comparación medias mediante ANOVA. Significación $p<0.05$. NS=No significativo. IPC=Inventario Procesos de Cambio.

TABLA 4. CORRELACIONES PROCESOS DE CAMBIO/HISTORIA DE CONSUMOY PSICOPATOLOGIA

\begin{tabular}{|c|c|c|c|c|c|c|}
\hline & & $\begin{array}{l}\text { AÑOS DE } \\
\text { CONSUMO }\end{array}$ & $\begin{array}{l}\text { AÑOS DE } \\
\text { ABUSO }\end{array}$ & BSI-GSI & BSI-PSDI & BSI-PST \\
\hline \multirow{5}{*}{$\begin{array}{l}\text { IPC PROCESOS } \\
\text { COGNITIVOS }\end{array}$} & Aumento de Concienciación & & & & & \\
\hline & Relieve Dramático & & & $\begin{array}{l}r=0.253 \\
p=0.015\end{array}$ & & $\begin{array}{l}r=0.311 \\
p=0.002\end{array}$ \\
\hline & Reevaluación Ambiental & & & & & \\
\hline & Autorreevaluación & & & $\begin{array}{l}r=0.304 \\
p=0.003\end{array}$ & $\begin{array}{l}r=0.251 \\
p=0.015\end{array}$ & $\begin{array}{l}r=0.342 \\
p=0.001\end{array}$ \\
\hline & Liberación social & $\begin{array}{l}r=0.208 \\
p=0.47\end{array}$ & & $\begin{array}{l}r=0.308 \\
p=0.003\end{array}$ & $\begin{array}{l}r=0.310 \\
p=0.002\end{array}$ & $\begin{array}{l}r=0.295 \\
p=0.004\end{array}$ \\
\hline \multirow{5}{*}{$\begin{array}{l}\text { IPC PROCESOS } \\
\text { CONDUCTUALES }\end{array}$} & Autoliberación & & & & & \\
\hline & Manejo de Contingencias & & & & & \\
\hline & Relaciones de Autoayuda & $p=0.033$ & $r=-0.223$ & & & \\
\hline & Contracondicionamiento & $\begin{array}{l}r=-0.362 \\
p=0.000\end{array}$ & $\begin{array}{l}r=-0.299 \\
p=0.004\end{array}$ & & & $\begin{array}{l}r=0.224 \\
p=0.31\end{array}$ \\
\hline & Control de Estímulos & & $\begin{array}{l}r=-0.223 \\
p=0.034\end{array}$ & & & \\
\hline
\end{tabular}

Correlaciones mediante coeficiente de correlación de Pearson.. IPC=Inventario Procesos de Cambio, BSI=Brief Symptom Inventory, GSI=Indice de Severidad Global, PSDI=Indice de Malestar. PST=Total Síntomas Positivos. No se incluye la variable "tratamientos anteriores" porque no muestra ninguna correlación. Sólo se han incluido los valores de correlaciones con significaciones inferiores a 0.05. 
ciona de forma positiva con los procesos liberación social, autoreevaluación y relieve dramático. El índice de malestar (PSDI) correlaciona también de forma positiva con los procesos liberación social y autoreevaluación. Finalmente el total de síntomas (PST) correlaciona positivamente con los procesos liberación social, autoreevaluación, contracondicionamiento y relieve dramático.

\section{DISCUSIÓN}

Los resultados de nuestro estudio confirman nuestras impresiones iniciales al encontrar distintos niveles de relación entre la presencia de psicopatología, el historial de uso y abuso de sustancias, y los distintos procesos de cambio por los que atraviesan nuestros pacientes.

Las puntuaciones medias en el inventario de procesos de cambio muestran que con independencia del diagnóstico de los pacientes todos utilizan con mayor frecuencia procesos de cambio de tipo cognitivo, en concreto los procesos reevaluación ambiental y autoliberación. Ello puede estar indicando por una parte que en el momento de demandar tratamiento, los pacientes han valorado el impacto que su comportamiento adictivo ha tenido en sus relaciones interpersonales y en las personas más allegadas, se produce mayor presión familiar y social y el sujeto reconoce las consecuencias negativas y las pérdidas que provoca el consumo. Por otra parte, parece producirse un compromiso personal para decidir la necesidad de cambio, y la creencia de que es el mismo sujeto el elemento esencial en dicho proceso al ser capaz de adquirir las habilidades necesarias para hacerlo, aparece la disposición al cambio comportamental y al estilo de vida.

En el extremo contrario observamos que también con independencia del diagnóstico, los procesos de cambio menos presentes en los pacientes al inicio del tratamiento son los procesos conductuales, en concreto los procesos de control de estímulos y contracondicionamiento. Ello podría estar indicando, que los pacientes se sienten incapaces de evitar la exposición a situaciones de alto riesgo para consumir y presentan dificultades para modificar sus respuestas elicitadas por estímulos condicionados, no pudiendo generar ni desarrollar conductas alternativas al consumo de la sustancia. Una baremación adecuada del instrumento podría proporcionar unos resultados más fiables.

Las relaciones encontradas entre los distintos procesos de cambio y las variables psicopatológicas y de consumo han sido variadas. Por un lado cuando los pacientes experimentan mayor nivel de distress emocional y más sintomatología existen más probabilidades de que experimenten las consecuencias negativas del consumo y la necesidad de pedir ayuda (relieve dramático), así como de percibir los beneficios de abandonar el consumo para que su vida se normalice (autoreevaluación). No obstante, los valores de las correlaciones son bajos, por lo que podría sugerirse la intervención de otro tipo de factores.

Los años de consumo, la presencia de sintomatología y la percepción subjetiva de malestar emocional aumenta la probabilidad de que el paciente se dé cuenta de que puede cambiar, se propicia una toma de conciencia de la posibilidad de cambio (liberación social). A mayor antigüedad en el consumo y más años de abuso de la sustancia se produce mayor deterioro psicosocial y menor existencia y menor utilización de los recursos de apoyo (relaciones de ayuda). Cuanto mayor es el historial de consumo se presentan más dificultades para modificar las respuestas condicionadas a la conducta adictiva, existe una mayor sensibilización a los efectos negativos de la abstinencia y menor capacidad para desarrollar conductas alternativas (contracondicionamiento). Un mayor historial de abuso provoca mayores dificultades para evitar situaciones de riesgo de consumo y, un mayor déficit en los mecanismos de control de impulsos (control de estímulos)

En general parece que la presencia de distress emocional y sintomatología influye en mayor medida en los procesos de tipo cognitivo, mientras que mayor historial de uso y abuso de la sustancia provocan mayores dificultades para adquirir y desarrollar estrategias conductuales de cambio. Sugerimos que la intervención psicoterapéutica al inicio de los tratamiento tendría que centrarse en la educación del paciente para el manejo de la sintomatología negativa y de distrés emocional y la utilización de técnicas de counseling, con el objetivo de proporcionar al paciente un confort psicológico que le permita orientar su estilo de vida hacia la abstinencia.

\section{REFERENCIAS BIBLIOGRÁFICAS}

Aragón, N. Bragado, C. Carrasco, I. (2000): Fiabilidad y estructura factorial del Inventario Breve de Síntomas (Brief Symptom Inventory) en adultos. Psicología Conductual, vol 8, n 1: 73-83

Asociación Psiquiátrica Americana (APA): (1995): Manual Diagnóstico y Estadístico de los Trastornos MentalesDSM-IV. Ed. Masson. Barcelona.

Bacon, M. (1975): The dependency-conflict hypothesis and the frecuency of drunkeers. J. Studies of Alcohol, 35: 863-876

Bell, R. (1975): Alcoholism life-events and psychiatric impairment. Sixth annual scientific conference national council of alcoholism. American Medical Society on alcoholism". Milwaukee.

Boulet, J Boss, M (1991): Reliability and validity of the Brief Symptom Inventory. Psychological Assesment, 3: 433437. 
Blum, E (1966): Psychoanalithic views if alcoholism. Quaterly Journal of Studies on Alcohol, 27 (259-299).

Cutter, H. (1973): Alcohol, power and inhibition. Q.J.Studies Alcohol, 17: 296-305.

Derogatis, L. (1975): Brief Symptom Inventory. Baltimore. Clinical Psychometric Research.

Derogatis, L. y Cleary, P. (1977): Confirmation of the dimensional structure of the SCL-90. A study in construct validation. J. Clin. Psychology 33(4): 981-989.

Derogatis, L.(1983): SCL-90-R. Administration, scoring and procedures manual. Baltimore. Clinical Psycometric Research.

DiClemente, C y Prochaska, J. (1985): Processes and stages of self-change: coping and competence in somoking behavior change. En Shiffman yWills (eds): Coping and substance use. Londres. Academic Press.

Jones, M. (1975): Social morbility and alcoholism: a comparison of alcoholics with their fathers and brothers. J. Studies of Alcohol, 36: 62-68.

Lettieri, D; Sayers, M; Pearson, H. (1980): Theories on drug abuse. Selected contemporany perspectives. national institute of drug abuse. U.S. Maryland.

Marlatt, A. (1985): Relapse prevention: theoretical rationale and overview model. En Marlatt, A. y Gordon, J. (eds): Relapse Prevention: Maintenance strategies in the treatment of addictive behaviors. New York. Guilford Press.

Piersma, H. Boes, J. Reaume, W. (1994): Unidimensional of the Brief Symptom Inventory in adult and adolescent patients. Journal of Personality Assessment, 63: 338344.
PNSD (Plan Nacional sobre Drogas) (1998): Observatorio español sobre drogas: Informe $n^{\circ} 1$. Madrid.

PNSD (Plan Nacional sobre Drogas) (1999): Observatorio español sobre drogas: Informe $n^{\circ} 2$. Madrid.

Prochaska, J. DiClemente, C. Norcross, J (1992): In sarch of how people change. Applications to addictive behaviors. American Psychologist, 47, 1102-1114.

Prochaska, j. y DiClemente, C. (1982): Transtheoretical therapy: toward a more integrative model of change. Psychotherapy: theory, research and practice, 19: 276278.

Prochaska, J. y DiClemente, C. (1984): Stages and processes of self-change of smoking: toward and integrative model of change. Journal of Consulting and Clinical Psychology, 51: 390-395.

Prochaska, J. y Prochaska J. (1993): Modelo transteorético de cambio para conductas adictivas. en: Recaída y prevención de recaídas. Ed. Neurociencias. Barcelona

Steiner, C. (1971): Games alcoholism play. Gower Press Inc. Baltimore

Tejero, A y Trujols, J. (1994): El modelo transteorético de Prochaska y Diclemente: un modelo dinámico de cambio en el ámbito de las conductas adictivas. En: Conductas Adictivas, J.L. Graña (ed). Ed. Debate. Madrid.

Tejero, A. Trujols, J y Hernandez, E. (1990): The process of change in heroin addicts: A preliminary report. Poster presentado al XX European Congress on Beaviour Therapy. París.

Vaillant, G. (1983): The natural history of alcoholism: causes, patterns and paths to recovery. Harvard University Press. 